\title{
Environment and the epochs of galaxy formation in the SDSS era
}

\author{
D. Thomas $^{1,2}$, C. Maraston ${ }^{1,2}$, K. Schawinski ${ }^{2}$, M. Sarzi ${ }^{3}$, S.-J. Joo ${ }^{4,2}$, \\ S. Kaviraj ${ }^{2}$ and S. K. Yi ${ }^{4}$ \\ ${ }^{1}$ Astrophysics, University of Oxford, Keble Road, Oxford, OX1 3RH, UK \\ ${ }^{2}$ Institute of Cosmology and Gravitation, Univ. of Portsmouth, Portsmouth, PO1 2EG, UK \\ ${ }^{3}$ Center for Astrophysics Research, University of Hertfordshire, UK \\ ${ }^{4}$ Department of Astronomy, Yonsei University, Seoul 120-749, Korea
}

\begin{abstract}
We analyse a sample of 1,652 elliptical galaxies morphologically selected from the SDSS DR4 with respect to their stellar population properties such as luminosity weighted ages, metallicities and $\alpha /$ Fe element abundance ratios. We confirm and statistically strengthen earlier results that all three stellar population parameters correlate with velocity dispersion, hence galaxy mass. Most surprisingly and differently from previous work, however, these scaling relations turn out to be independent of environmental density for the bulk of the population. This implies that the formation epochs and timescales of the stellar populations in massive galaxies are entirely independent of the environmental density and only driven by galaxy mass. At the low-mass end some fraction of the galaxies show signs of recent star formation on top of the general downsizing trend. It is only the fractional amount of such rejuvenated galaxies that reveals strong dependence with environment. The influence of the environment apparently undergoes a phase transition at about $z \sim 1$. An early galaxy formation phase without environmental dependence is followed by a rejuvenation phase at redshifts below $z \sim 1$ affecting mostly low-mass galaxies, during which the environment plays a crucial role.
\end{abstract}

Keywords. stars: abundances, Galaxy: abundances, galaxies: stellar content, galaxies: elliptical and lenticular, $\mathrm{cD}$, galaxies: evolution, galaxies: formation

\section{Introduction}

The environment is known to affect considerably the formation and evolution of galaxies. Its influence is best visible through the well-known morphology-density relationship, according to which early-type galaxies and morphologically undisturbed galaxies are preferentially found in high density environments and vice versa (Oemler 1974; Dressler 1980).

In contrast, it is not clear at all whether the environment is equally important at a given galaxy morphology. There is still major controversy about whether the formation and evolution of the most massive and morphologically most regular galaxies in the universe, i.e. elliptical galaxies, are significantly affected by environmental densities. A general difficulty is to define samples that distinguish properly the various environmental densities. The Sloan Digital Sky Survey (SDSS) provides the opportunity to overcome this limitation. It allows to investigate, for the first time, huge samples of local earlytype galaxies, so that a statistically meaningful investigation of the stellar population parameters of galaxies and their dependence on environment can be attempted.

In this paper we discuss the stellar population properties of a sample of elliptical galaxies drawn from the SDSS DR4. We infer the formation epochs of early-type galaxies as a function of galaxy mass and environmental density. This work is an extension of 
Thomas et al. (2005, hereafter T05), with the major improvement of increasing the sample size by more than an order of magnitude.

\section{Data catalogue}

We have collected a magnitude limited sample of 1,652 elliptical galaxies in the redshift range $0.05 \leqslant z \leqslant 0.055$ with apparent $r$-band magnitude brighter than 16.8 from the SDSS Data Release 4. The most radical difference with respect to other galaxy samples constructed from SDSS is our choice of pure morphological selection of galaxy type by visual inspection. For the estimate of the environmental density we adopt the method developed and described in full detail in Schawinski et al. (2007). As a result we obtain the 3-dimensional volume density at the location of each object, hence an estimate of the local environmental density. The kinematics of gas and stars are determined using an improved version of the the method of Sarzi et al. (2006, see also Sarzi's contribution to this proceedings for details). An important improvements in this approach is that stellar population and emission line templates are fitted simultaneously to the galaxy spectrum. On each spectrum, we then measure the 25 standard Lick absorption line indices following the index definitions of Trager et al. (1998). The stellar population models of Thomas et al. $(2003,2004)$ are used to derive luminosity-weighted ages, metallicities, and $\alpha / \mathrm{Fe}$ ratios by minimising the $\chi^{2}$ of the fit to all 25 indices. A detailed description of the catalogue can be found in Schawinski et al. (in preparation), and we refer the reader to this paper for any details.

\section{The stellar population scaling relations}

Despite the general agreement in the recent literature on the existence of an age-mass relationship within the early-type galaxy population, there is some debate about the exact slope. Several recent studies (e.g., Nelan et al. 2005; Bernardi et al. 2006) infer a significantly steeper slope than T05. As discussed by Renzini (2006), this relatively steep slope implies strong rotation of the Fundamental Plane as a function of redshifts, that is inconsistent with findings of intermediate-redshift studies. The major difference between T05 and other work in the literature is the treatment of the low-mass end of the early-type galaxy mass function. The emergence of an increasing fraction of rejuvenated galaxies at lower masses (T05; Yi et al. 2005; Thomas \& Davies 2006; Schawinski et al. 2007) considerably steepens the simple linear fit. As these young low-mass ellipticals are not representative of the bulk population, they are excluded from the fit in T05. The very large and homogeneous data set we have at hand in the present work allows for a more detailed investigation of this issue.

The left-hand figure in Fig. 1 shows the correlation between luminosity-weighted age and velocity dispersion for various environmental densities decreasing from bottom to top. The ages of the bulk population (black symbols) exhibit an approximately Gaussianlike distribution about the fit applied to the entire sample (black lines and histograms). Some fraction of rejuvenated galaxies (grey symbols) leads to a tail in the distribution to younger ages (grey histograms).

Most importantly, the positions of the black histograms in the right-hand panels do not differ significantly. This implies that the age- $\sigma$ relationship is independent of the environment for the bulk of the population. Very different, however, is the distribution of the tail population. The fraction of objects in the young tail population is negligible in the densest environments and increases to about 40 per cent at the lowest densities. Hence, ellipticals in lower density environments are not generally younger (at a given 

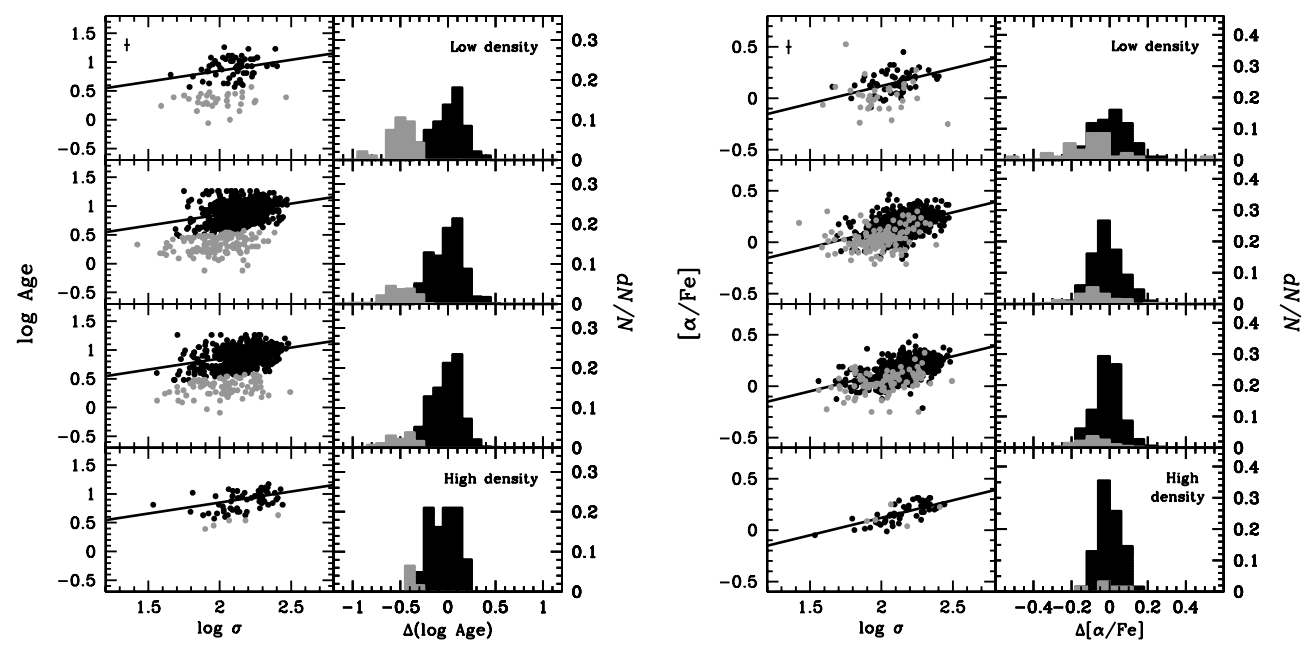

Figure 1. Left-hand figure: Luminosity-weighted age as a function of velocity dispersion. Black circles are the bulk population following a Gaussian distribution, grey circles are the rejuvenated tail population. Different panels imply different environments with environmental density increasing from top to bottom. The solid line is the linear fit to the bulk population (black circles) without environment separation. Right-hand panels: Deviation from the linear fit for the bulk population (black histogram) and tail population (grey histogram). Right-hand figure: Same for element abundance ratio $\alpha / \mathrm{Fe}$.

mass), but the fraction of rejuvenated objects increases with decreasing environmental density.

The right-hand figure of Fig. 1 shows the $\alpha / \mathrm{Fe}-\sigma$ relationship, which, again, is independent of the environmental density for the bulk of the population. The rejuvenated objects discussed above exhibit relatively low $\alpha / \mathrm{Fe}$ ratios at a given $\sigma$. This confirms that rejuvenation owing to late star formation results in significant enrichment of $\mathrm{Fe}$ from Type Ia supernovae.

\section{The formation epochs}

Following the approach of T05, the luminosity-weighted ages and $\alpha /$ Fe element ratios derived here enable us to approximate star formation histories. The result is shown in Fig. 2, which illustrates the key observation that the stellar populations of more massive galaxies form faster and at higher redshift, now commonly referred to as 'downsizing'. A fundamental difference with respect to previous results is the dependence on environment. The main formation epochs of elliptical galaxies are independent of the environmental density.

We recall that the fraction of rejuvenated galaxies, instead, is crucially dependent on environment. The importance of this process increases with decreasing galaxy mass, and is negligible in the most massive objects (see Fig. 1). Such rejuvenation is expected to occur in the past few billion years (Thomas \& Davies 2006), clearly below redshift $z \sim 1$. This implies the occurrence of a phase transition at that redshift as indicated in Fig. 2. At very early epochs the formation and evolution of galaxies is driven solely by intrinsic galaxy properties such as mass and is independent of the environmental densities. This stage is then followed by a rejuvenation phase at redshifts below $z \sim 1$ affecting mostly low- and intermediate mass galaxies, during which the environment, hence galaxy interactions, play a crucial role. 


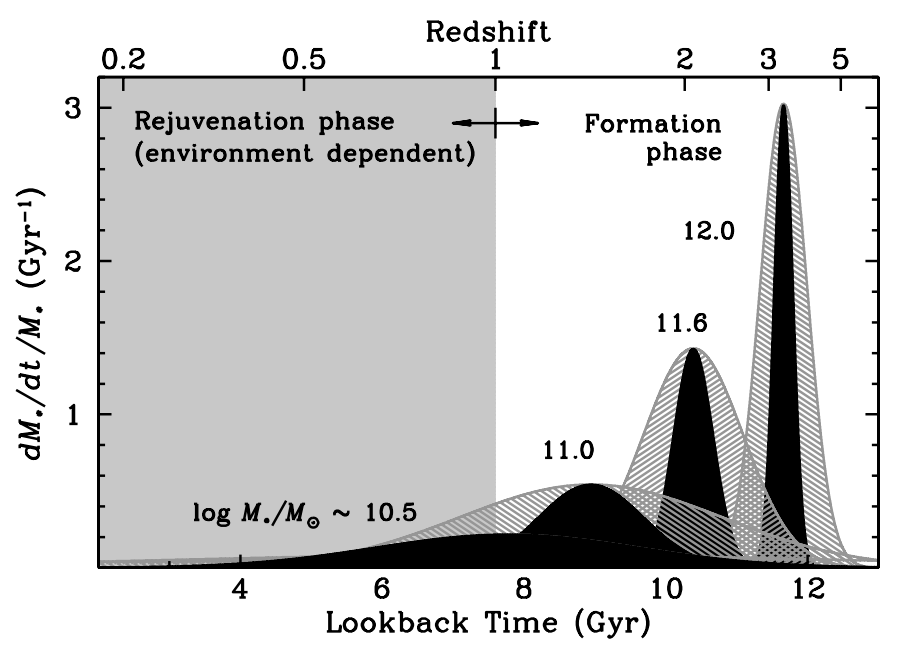

Figure 2. Specific star formation rate as function of look-back time for elliptical galaxies of various masses as indicated by the labels. The grey hatched curves indicate the range of possible variation in the formation timescales that are allowed within the intrinsic scatter of the $\alpha / \mathrm{Fe}$ ratios derived. No dependence on environmental density is found. The upper x-axis connects time and redshift adopting $\Omega_{m}=0.24, \Omega_{\Lambda}=0.76$, and $H_{0}=73 \mathrm{~km} / \mathrm{s} / \mathrm{Mpc}$ (Tegmark et al. 2006).

With this picture we refine the conclusion of Kormendy \& Kennicutt (2004), that currently 'the universe is in transition' from a merging dominated epoch in the past to a secular evolution phase in the far future. By adding the phase transition at $z \sim 1$ we divide the first, merger dominated phase of the Kormendy \& Kennicutt picture in two halves. Eventually, the Universe may then return to a time in which intrinsic galaxy properties re-gain dominance over galaxy interactions as the main driver for galaxy formation.

\section{Acknowledgements}

DT acknowledges support by grant BMBF-LPD 9901/8-111 of the Deutsche Akademie der Naturforscher Leopoldina.

\section{References}

Bernardi, M., Nichol, R. C., Sheth, R. K., Miller, C. J., \& Brinkmann, J., 2006, AJ, 131, 1288

Dressler, A., 1980, ApJ, 236, 351

Kormendy, J., \& Kennicutt, R. C., 2004, ARA\&A, 42, 603

Nelan, J. E., et al., 2005, ApJ, 632, 137

Oemler, A. J., 1974, ApJ, 194, 1

Renzini, A., 2006, ARA\&A, 44, 141

Sarzi, M., et al., 2006, MNRAS, 366, 1151

Schawinski, K., et al., 2007, ApJ, in press, astro-ph/0601036

Tegmark, M., et al., 2006, Phys. Rev. D, in press, astro-ph/0608632

Thomas, D., \& Davies, R. L., 2006, MNRAS, 366, 510

Thomas, D., Maraston, C., \& Bender, R., 2003, MNRAS, 339, 897

Thomas, D., Maraston, C., Bender, R., \& Mendes de Oliveira, C., 2005, ApJ, 621, 673

Thomas, D., Maraston, C., \& Korn, A., 2004, MNRAS, 351, L19

Trager, S. C., Worthey, G., Faber, S. M., Burstein, D., \& González J. J., 1998, ApJS, 116, 1

Yi, S. K., et al., 2005, ApJ, 619, L111 


\section{Discussion}

RENZINI: It is perhaps worth mentioning that just $10 \%$ of baryons are now in stars, both in clusters of galaxies as well in the general field. Once more, at this top level environment seems to be irrelevant.

Thomas: I agree.

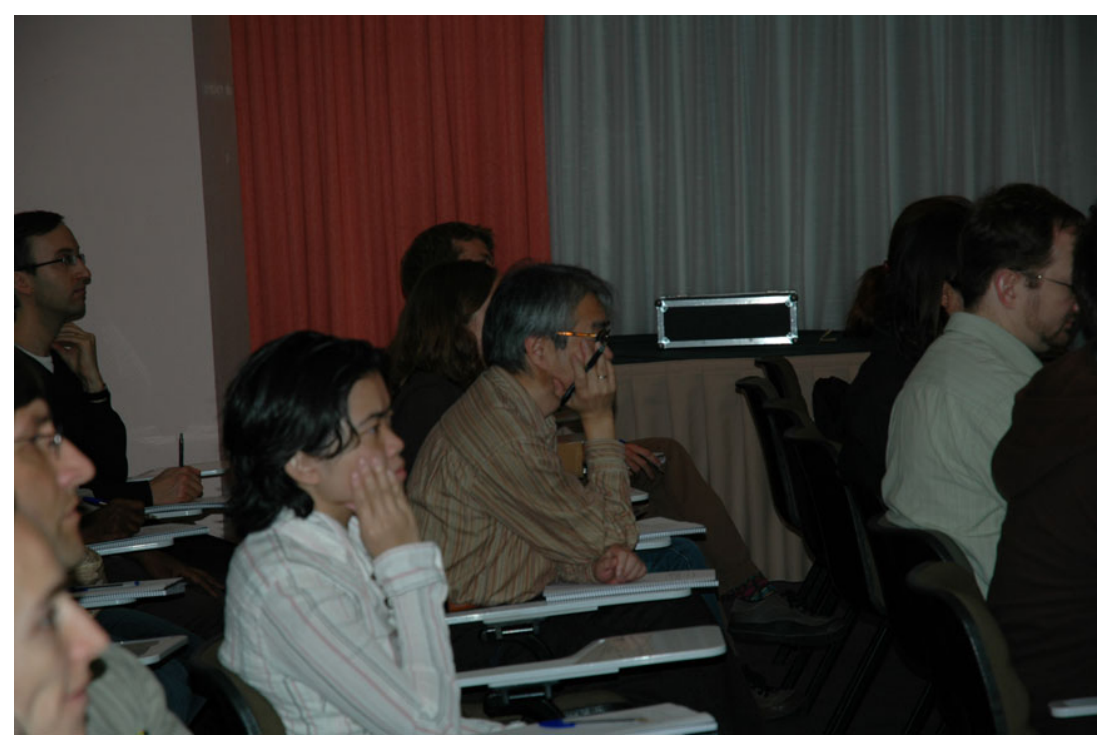

Ching-Wa Yip (foreground) and Nobuo Arimoto (middle).

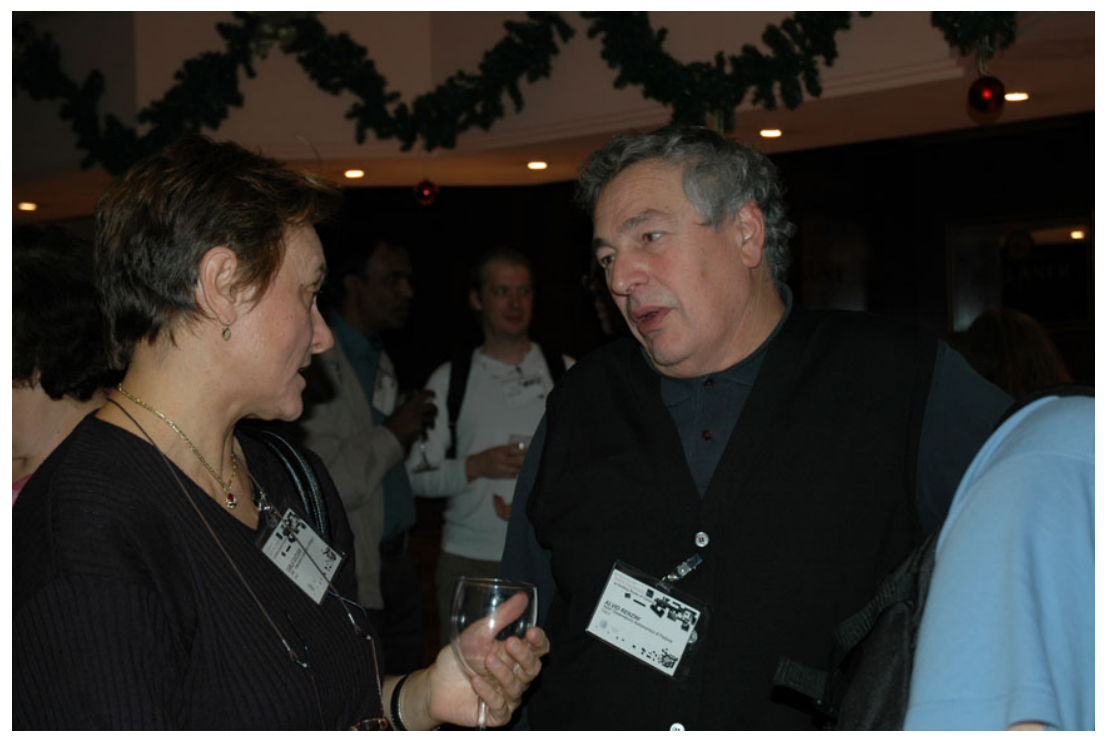

Carla Cacciari (left) speaking to Alvio Renzini. 